\title{
A Finite Element Modeling and Simulation of Human Temporomandibular Joint with and Without TM Disorders: An Indian Experience
}

\author{
Mehak Sharma*, Manoj Soni \\ Department of Mechanical and Automation Engineering, Indira Gandhi Delhi Technical University for Women, New Delhi \\ 110006, India
}

Corresponding Author Email: er.mehaksharma@gmail.com

https://doi.org/10.18280/mmep.080303

Received: 16 January 2020

Accepted: 5 December 2020

\section{Keywords:}

finite element analysis, TMJ, jaw joint, biomechanics, stress distribution, bruxism, clenching

\begin{abstract}
Temporomandibular joint (TMJ) is anatomically the most intricate joint which connects the lower jaw to the upper jaw and regulates jaw movements. It significantly deals with mastication and speech. It is hence imperative to study the mechanics and functioning of the jaw joint to devise alternative solutions for its replacement whenever required. Further, human skulls are anthropologically categorized into three types - African, Asian and European. Out of these, the Indian skull is also a bit different than its Asian counterparts because of its osteology and skeletal biology. Hence, a comprehensive biomechanical and computational study is essential to provide customized solutions. For the present study, four different loading conditions are selected to perform finite element analysis on the human skull, Anonymized and unidentifiable CT scan data sets from open-source web platforms are converted to STL and then 3D models using 3D slicer. Finite element analysis of jaw joint is carried out. Results based on Von Mises stress studies show significant behavioral differences under varying load conditions. Hence, it is crucial to identify solutions for TMJ disorders of the Indian population.
\end{abstract}

\section{INTRODUCTION}

The temporomandibular joint is both ginglymus (hinging joint) and arthrodial (sliding joint), which is why it is often labeled as ginglymoarthrodial joint as shown in Figure 1. It is an extensively used joint in the human skull and body. TMJ opens and closes numerous times daily and is vital for speech, snoring, mastication, deglutition, yawning and the involuntary alliance of articular pairs [1].

Since a multitude of Indian population is suffering from TMJ disorders, therefore it is critical to address TMJ issues taking all considerable frames of reference. There is paucity in understanding connections among muscle behavior, jaw motions, craniofacial morphology and all the forces acting on TMJ. Extensive research and awareness are needed to understand the etiology of temporomandibular joint disorders found in Indian patients. New inventions and methods should be adopted and followed to analyze, avert, and alleviate these disorders.

The analysis of mandibular biomechanics helps us understand the mechanism, activity and pattern of temporomandibular disorders can be presumed by analyzing the TMJ biomechanics. This will aid in up-gradation in the design and performance of prosthetic devices, thereby enhancing the serviceable life of these devices. TMJ prosthetic replacement is an extensive surgery and it is only advisable as last resort. Testings and experiments on TMJs of actual human beings are also avoided because administering experimental devices inside the TMJ can cause severe harm to its tissue. Therefore researchers, surgeons, diagnosticians and scientists use in-vitro techniques such as CT scanning, laser 3D scanning, finite element analysis (FEA) and other analytical technologies for extensive research in TMJ biomechanics.

Spencer and Demes discussed in a classic study of basic joint mechanics to assess the differences in size and shapes of jaw mandible and it is also [2] useful in understanding the bite forces for different generations and races. Chen and $\mathrm{Xu}$ discussed and constituted a 2-D FE model of human TMJ which deduced the distribution of stresses [3] between condylar, temporal surfaces and articular disk. Finite element analysis has been in use for the study of the biomechanics of joint since the 70s, Richmond et al. successfully incorporated the morphology and biomechanical [4-9] properties of bone, muscles and tissues in constructing and improving FEA models that can calculate the stresses and strains. On the other hand, Kober et al., Strait et al., Ren et al., and Milne et al. [1013] demonstrated various errors arising in substantially depicting the anatomy, internal structure, mechanisms, muscle forces, loads and spatial constraints using FEA technique. Richmond et al. successfully incorporated the morphology and biomechanical [14] properties of bone, muscles and tissues in constructing and improving FEA models that can calculate the stresses and strains. There is a relative certainty that FEA is a vital tool for studying the biomechanics of TMJ, keeping the evidence of errors alongside [15-20]. Aoun et al., presented a hypothesis (also used in the current study) to elaborate the functioning of TMJ in three static [17] mandible positions by clenching using FEA models and thus the behavior of natural TMJ has been characterized using computational analysis.

Dimitroulis, an Australian medical pioneer in the field of study of Temporomandibular joint has narrowly discussed the vitality of extensive studies to understand TMJ, its functioning, mechanisms, disorders, diseases, solutions, replacements, surgical needs and the whole rationale in the Indian context. 
[21] It has been predicted that Indian Maxillofacial specialists need to be prepared for recognizing and managing disorders that present with more complex symptomatology where the aspect of TMJ surgery is extremely viable.

Ingawalé and Goswami explained and discussed the temporomandibular joint anatomy [22] and its biomechanics (Indian origin TMJ included) in the book titled Human Musculoskeletal Biomechanics, it extensively details the various methods and techniques as well as hypotheses utilized in the study of the biomechanics of human TMJ. To correctively examine the type and range of TMJ disorder, FEA was carried out simulating the response of TMJs exhibiting a restrictive condylar displacement during the opening and closing movement by Creuillot et al. [23] validating the importance of articular disc and functional ligaments in natural motions of TMJ. There are many other instances in literature [24-28] where FEA was conducted to determine stresses at various mandible positions and for various loading conditions too.

Ndukwe and Anitha [29] studied the scope, application analysis and also limitations of 3D models for ascertaining the use of FEA in biomechanical analysis of human jaw joint. THE human TMJ disc is the most significant part of TMJ anatomy which is being studied by Gomes et al. to establish the potential use of tissue engineering in the TMJ domain for substituting articular disc. Pre-clinical trials involving FEA experiments [30-32] have been carried out to evaluate potential biomaterials and to increase knowledge regarding disease mechanisms. An archaeological point of view has been presented by Stansfield et al. [33] which suggested a sensible approach in future comparative studies using FEA techniques for biomechanical studies of the human joint. It has been put forward in the study that accurate modeling of a human skull is unattainable and conclusions from computational analysis are subjective to the sensitivity of input parameters.
Summarizing the literature, it has been observed that there are numerous studies dedicated to exploring the behavioral differences in TMJ with normal functioning and TMJ with disorders in the medical and scientific arena. But when there arises a need for customized last resort solutions such as prosthesis or total joint replacement, there is a dearth of information on stresses acting on TMJ or jaw joints of the Indian population. There is also a lack of research in finite element analysis or in-vitro experiments for zeroing in on the factors responsible for TMJ disorders, specifically for the Indian population.

In the present paper, our objective as derived after careful literature review is to investigate jaw joints of Indian ethnicity with and without disorders using the finite element analysis method. The variations in boundary conditions and material properties are specified sensitively to the FE model as discussed in section 2.1. CT scan data sets (anonymized and unidentifiable) are used to create an STL file and 3D finite element models. Von mises stress developed under varying loading conditions and are thereafter being compared with values as obtained from other literature studies.

It has been found that computational analysis performs steadily in predicting stresses of a model of human TMJ. The approach to achieve the aforementioned conclusion involves the in-vitro experimentation under four loading conditions balanced occlusion, unbalanced occlusion, bruxism and clenching.

It can also be presumed that the performance models of these jaw joints (specific to Indian ethnicity) obtained from open-source web platforms may vary significantly from the others in the world. This presumption can form the basis of future work which involves customized designing of TMJ prosthesis specific to Indian patients suffering from TMJ disorders.

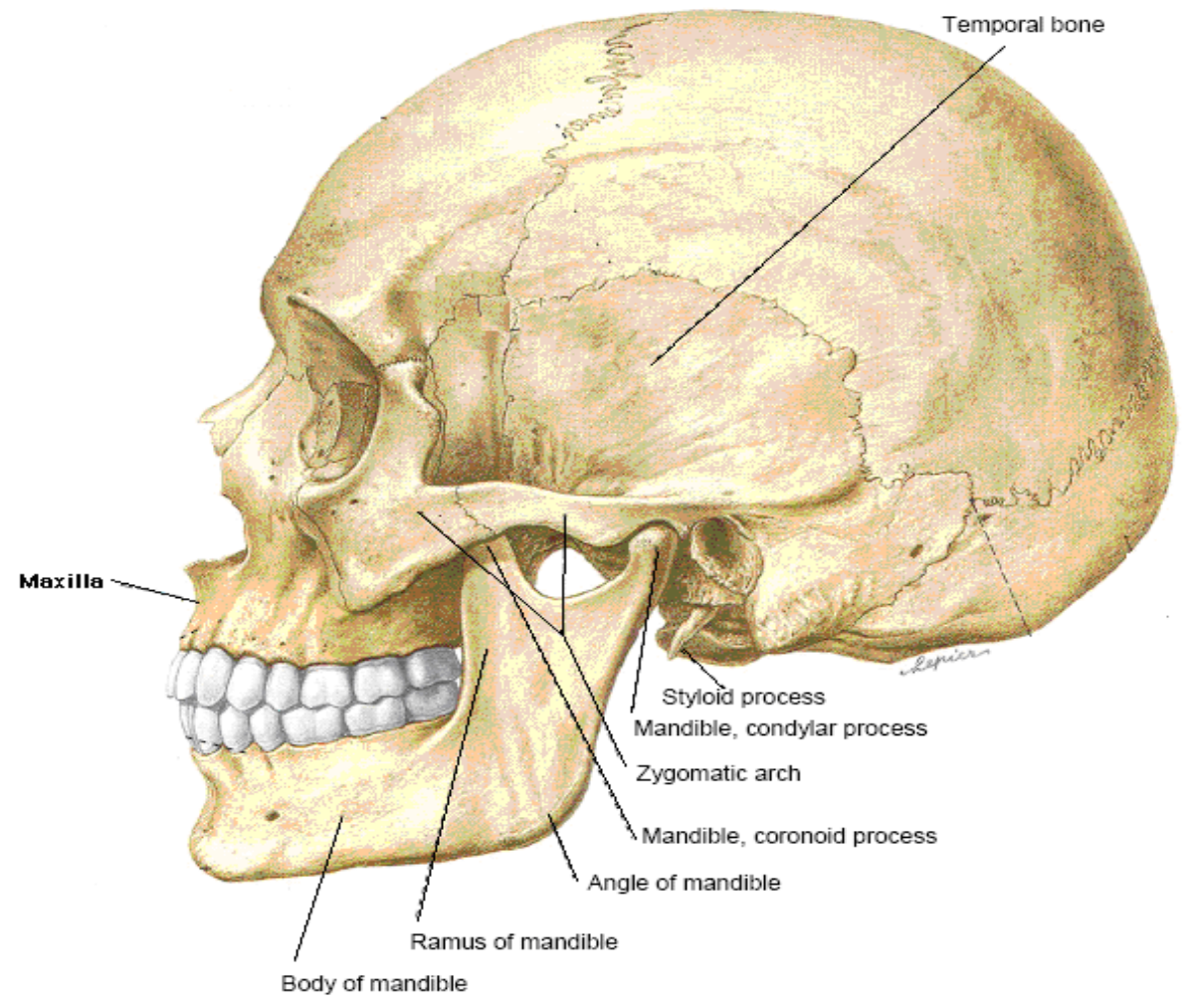

Figure 1. Human jaw anatomy [1] 


\section{MATERIALS AND METHODS}

CT scan data sets are acquired from open-source platforms like 3D Slicer, DICOM Library, Osirix keeping the data sets unidentified and anonymized. One CT scan is with no abnormalities and the other CT scan is with a degenerative disorder (ankylosis). The contours of the cranium and the mandible along with the lower jaw, articular fossa and teeth are obtained from CT images. The soft tissue contours for the articular disc and muscles in connection with bony elements of TMJ are located with assistance from an oral and maxillofacial professional. Bones are rather inflexible as compared to the relevant soft tissues hence they are classified as a rigid material. The STL of all bone components are created in a 3D slicer and then are reconstructed/meshed automatically in ANSYS analysis software.

The soft tissue contours of the joint and the articular disc are created and meshed in the software. The material properties are assigned to the bony as well as soft tissue components in the finite element framework from the literature. The finite element meshes of the temporal bone, mandible, articular disc and ligaments are constructed in ANSYS using eight-node brick elements.

Few contact pairs are established to define the relationship between articular disc, mandible, condyle, fossa, temporal bone, and ligaments of the interaction between them. The articular disc is in direct contact with the temporal bone and condyle. The temporomandibular ligament is connected with the jaw and with the lateral part of the disc. Since the synovial fluid is present inside the mouth, a friction coefficient of 0.0001 is considered for all interactions. As per our literature review and analysis, Koolstra has explained that the most detrimental factor for internal joint [19] disorders is high friction due to less lubrication in the jaw joint. In this article, we have analyzed a human joint with a dislocated articular disc and it is a very common internal joint disorder known as ankylosis.

All these conditions are true to the data collected from CT scan with no abnormalities whereas, for CT scan data with a degenerative disorder, the temporal bone and condyle are fused due to the absence of articular disc between them. Hence two sets of analyses are conducted for four different boundary conditions between disc and bone: i) Normal disc movement, (coefficient of friction 0.0001), free sliding; and ii) Fused jaw bone without any sliding, penetration or separation. The boundary conditions are taken from the literature [22].

\subsection{Boundary and load conditions}

The functioning of TMJ can be understood by predicting the stresses occurring on the mandible, the disc and the joint for different bite forces and various bite patterns. Each force vector shown in Figure 2 represents various existing muscle forces and is determined according to different muscular regions and patterns. These force vectors are distributed and assigned values to obtain the desired resultant force for varying boundary conditions. The mesh models for the normal and fused jaw are imported to ANSYS to study stress distribution and its outcomes in the TMJ to test four different hypotheses. Considering bone to be a ductile material, Von Mises failure criterion is used to investigate the stress profile of TMJ. The models are given linear and isotropic properties from literature (Table 1).

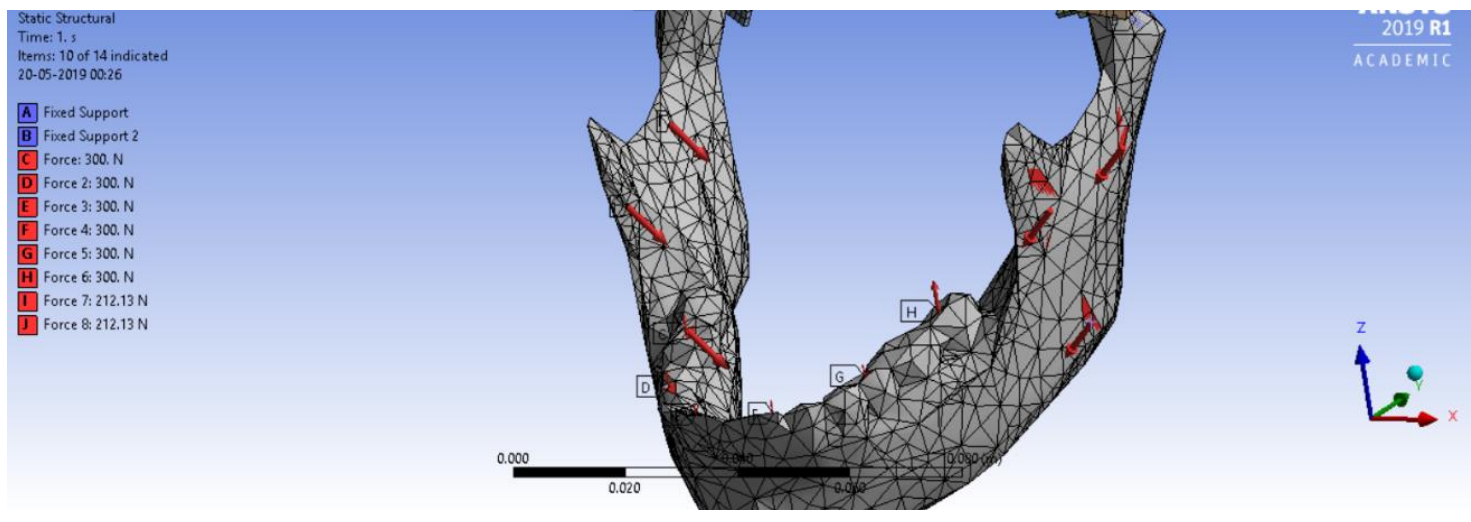

Figure 2. Boundary loading conditions representing forces acting on the FE model

Table 1. Isotropic properties for material in the FE mesh model

\begin{tabular}{ccc}
\hline Material & Young's Modulus (GPa) & Poisson's Ratio \\
\hline Cortical bone & 17 & 0.3 \\
Teeth & 50 & 0.25 \\
Cancellous bone & 0.5 & 0.3 \\
Cartilage & 0.006 & 0.49
\end{tabular}

The articular fossa is considered as fixed support and the condyle is allowed to have anterior-posterior linear motion as well as rotation. Resultant force values (Table 2) are assigned from Ingawale et al. [22]. For different loading conditions, maximum Von Mises Stress is found at the condylar head. For both normal and fused TMJs, balanced occlusion showed the least values for maximum Von Mises stresses.
Table 2. Resultant Forces applied to FE mesh models, with the location of max stresses

\begin{tabular}{|c|c|c|c|c|}
\hline \multirow{2}{*}{$\begin{array}{c}\text { Load } \\
\text { Condition }\end{array}$} & \multicolumn{2}{|c|}{ Resultant Force (N) } & \multirow{2}{*}{\multicolumn{2}{|c|}{$\begin{array}{c}\text { Location of } \\
\text { Maximum Von Mises } \\
\text { Stress } \\
\end{array}$}} \\
\hline & Left & Right & & \\
\hline Balanced & 200 & 200 & \multicolumn{2}{|c|}{ Equal on both condyle } \\
\hline Unbalanced & 250 & 400 & \multicolumn{2}{|c|}{ Right condyle } \\
\hline \multirow[t]{2}{*}{ Bruxism } & $\begin{array}{c}400 \\
\text { (vertical) }\end{array}$ & $\begin{array}{c}500 \\
\text { (vertical) }\end{array}$ & \multirow{2}{*}{\multicolumn{2}{|c|}{ Left Condyle }} \\
\hline & \multicolumn{2}{|c|}{300 (transverse) } & & \\
\hline Clenching & 1000 & 1000 & Equal & $\begin{array}{c}\text { Right } \\
\text { Condyle }\end{array}$ \\
\hline
\end{tabular}

\subsection{Types of loading}

Biomechanical behavior of TMJ is studied for two different 
scenarios: a) Normal jaw and b) Fused jaw. The different loading conditions along with isotropic properties are detailed in Table 1 and Table 2 respectively. All these models are discussed below concerning each loading condition.

\subsubsection{Balanced occlusion}

For a balanced loading condition, the 3D mesh model obtained from CT scan data for normally functioning TMJ has been assigned material properties as discussed previously. The model is refined, a volume mesh generated and then investigated for comparative stress in the joint for $200 \mathrm{~N}$ load (Table 2) on both the left and right side of the jaw. The model is constrained to imitate the movements of the jaw during normal opening and closing. During balanced occlusion, both condyles can linearly move in the anterior-posterior direction along with few degrees of rotation in the mediolateral axis.

Same constraints are applied and simulated for the mesh model of the human jaw with displaced disc or fused jaw.

\subsubsection{Unbalanced loading}

For unbalanced loading condition, the mesh model for both normal and fused TMJs is subjected to unbalanced loading (Table 2). The left side of the jaw is assigned a load of $250 \mathrm{~N}$ and the right side is assigned a $400 \mathrm{~N}$ load. Same constraints for linear and rotational movements assigned for balanced occlusion has been applied for unbalanced occlusive loading.

\subsubsection{Bruxism}

In the case of bruxism, mesh models are subjected to distinctive loads in vertical and transverse directions. Also, the left side of the jaw has been assigned a different load as compared to the right side. The values are given in Table 2 . During bruxism, the forces are applied on the first and second molars, second premolar on both sides. All the conditions and constraints are the same for a normally functioning jaw as well as a fused jaw.

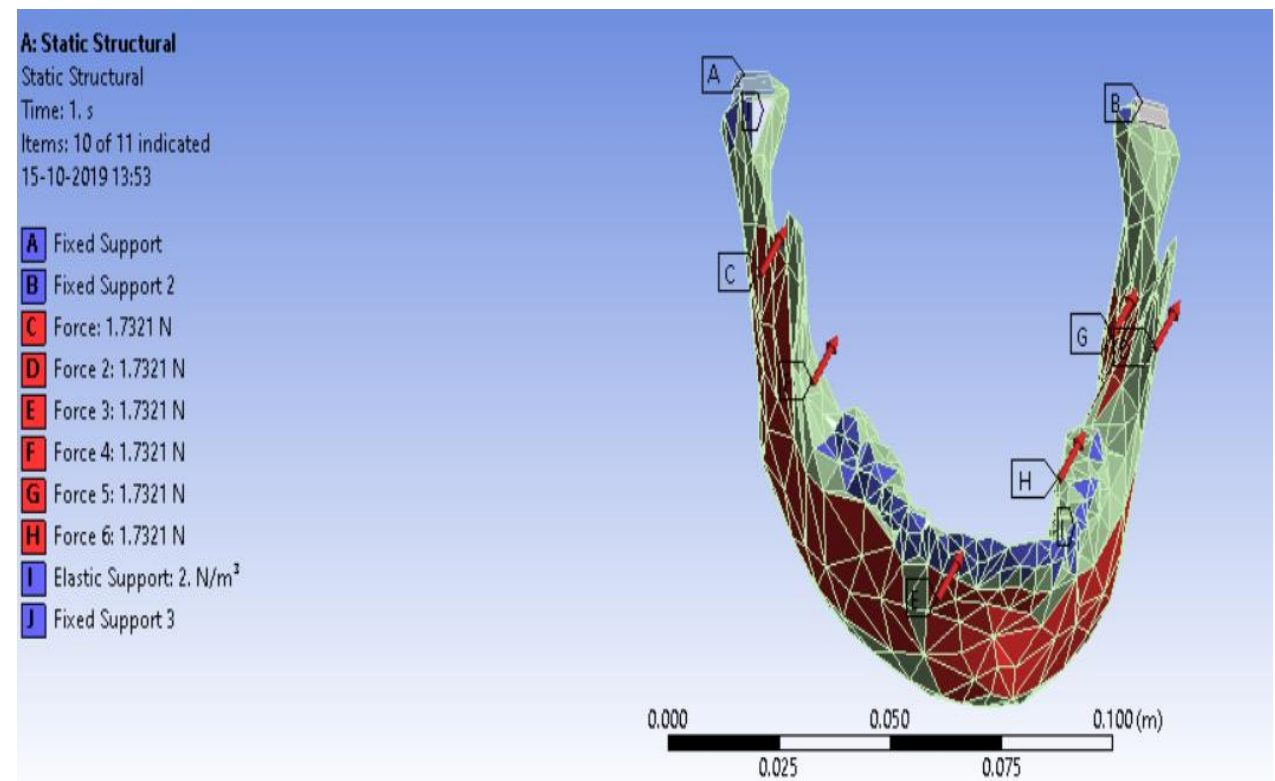

Figure 3. Balanced load $200 \mathrm{~N}$

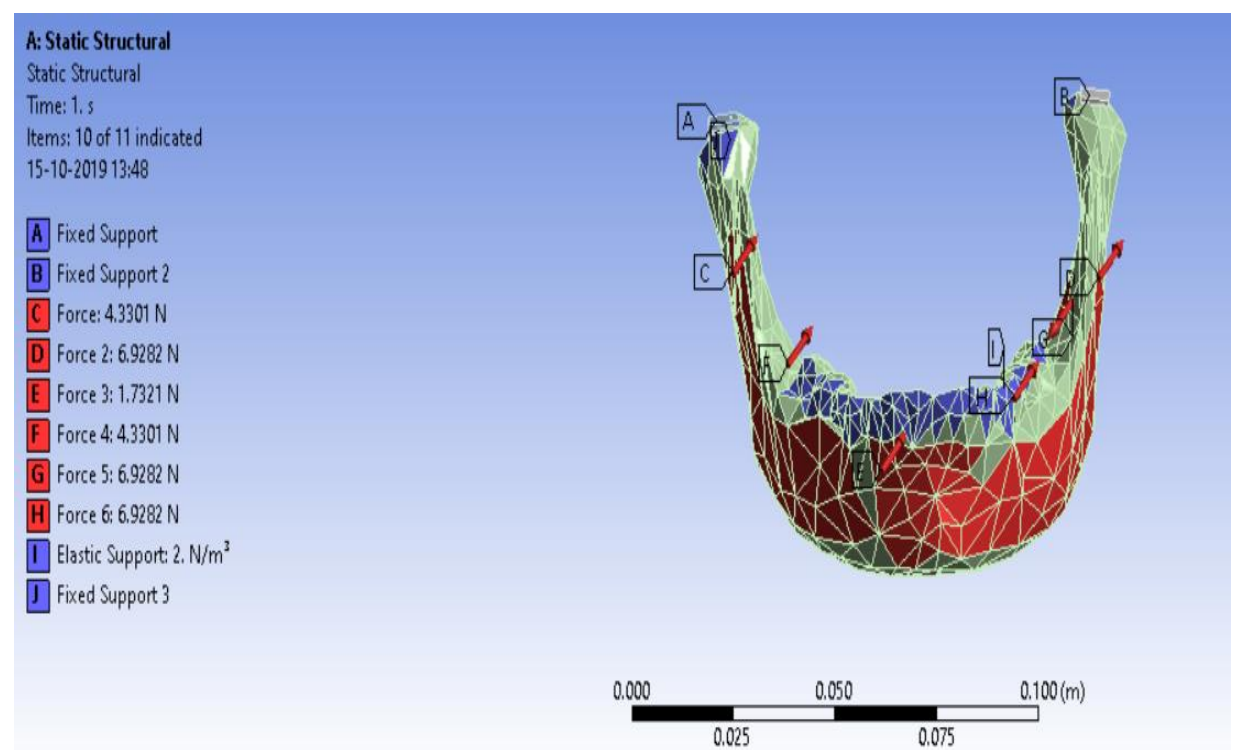

Figure 4. Unbalanced load of $250 \mathrm{~N}$ and $400 \mathrm{~N}$ 


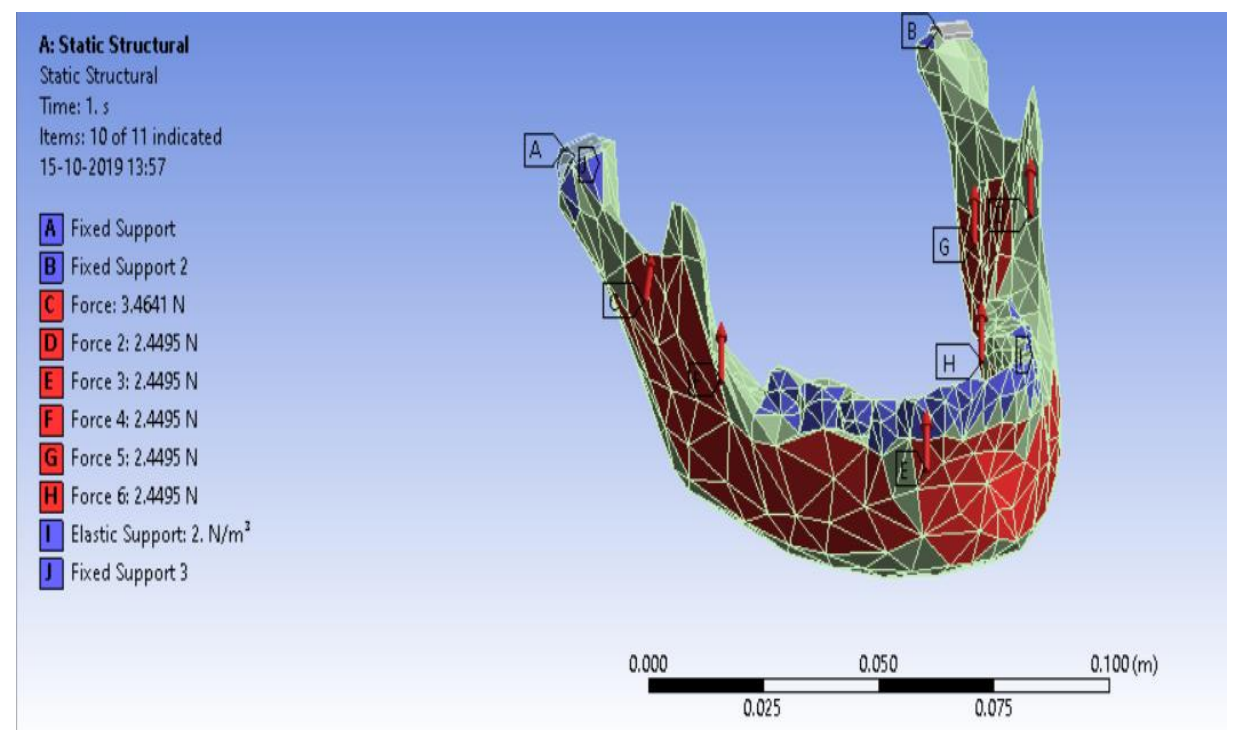

Figure 5. Teeth grinding or bruxism

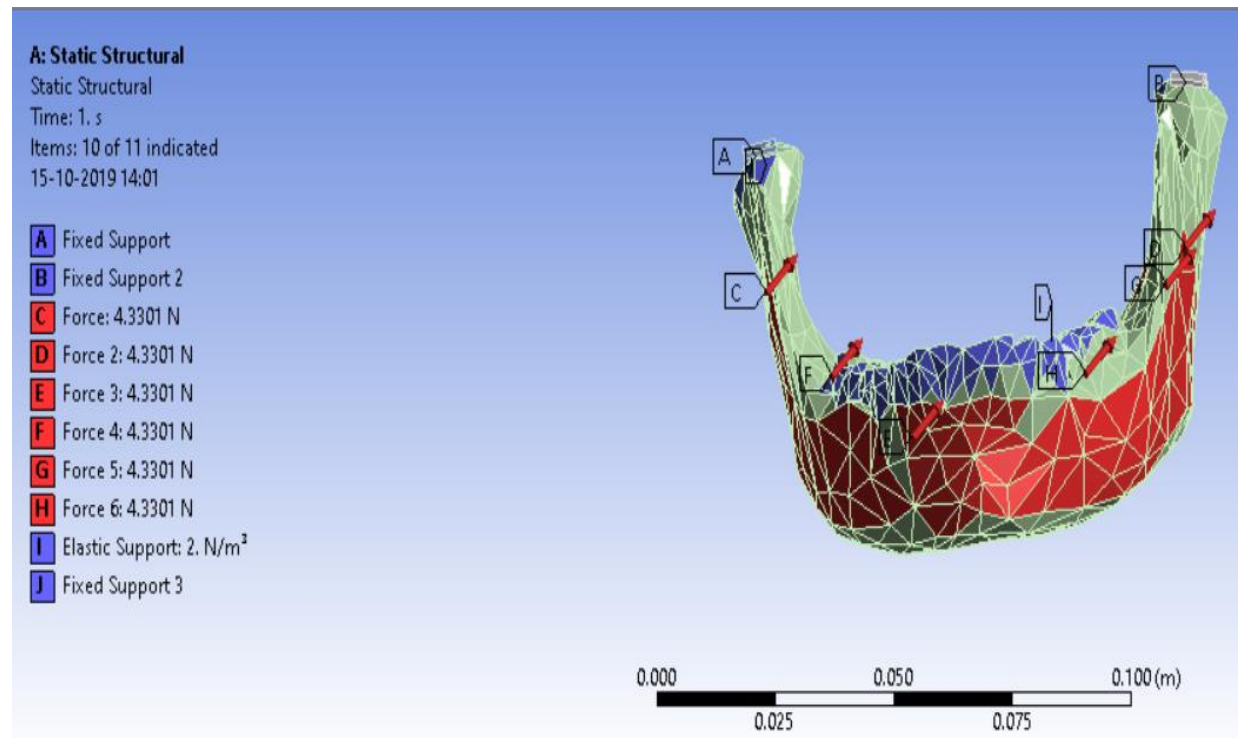

Figure 6. Clenching $1000 \mathrm{~N}$

\subsubsection{Clenching}

The simulation of bilateral molar clenching has been performed to investigate the comparative stress development and distribution in the normal and fused jaw. A $1000 \mathrm{~N}$ load has been applied to both sides in case of a normally functioning jaw, with linear and rotational movements as that of balanced occlusion. For a fused jaw, the load of $1000 \mathrm{~N}$ has been applied on the first and second molars and the second premolar on the right side only, keeping the articular surface constrained.

It has to be considered that the magnitudes of TMJ forces differ significantly in literature and there are no universally accepted values among researchers on TMJ loading [22]. Due to the presence of conflicting views on the magnitude, type and direction of TMJ forces in literature, researchers use different TMJ loading conditions for finite element modeling.

\subsection{Analyses}

For normal and deformed models, four varying load conditions are formulated as shown in Figures 3-6. Figure 3 represents balanced load, 4 represents unbalanced, 5 represents bruxism and 6 represents clenching respectively. Analyses are carried out to investigate Von Mises stress distribution for each loading condition.

\section{RESULTS AND DISCUSSION}

\subsection{Results}

Figures 7 to 9 present Von Mises stress distribution for the normal jaw and fused jaw for four loading conditions i.e., Balanced occlusion (min force $200 \mathrm{~N}$ ), Unbalanced occlusion, Bruxism and Clenching ( $\max$ force $1000 \mathrm{~N}$ ) in FEA simulation. The results have been validated with the available literature for normal jaw. For a fused jaw, FEA simulation for varying load conditions has been attempted for the first time to our knowledge for the Indian populace. Comparison is drawn between the maximum value of Von Mises stress for normal and fused jaw for different types of occlusions. 


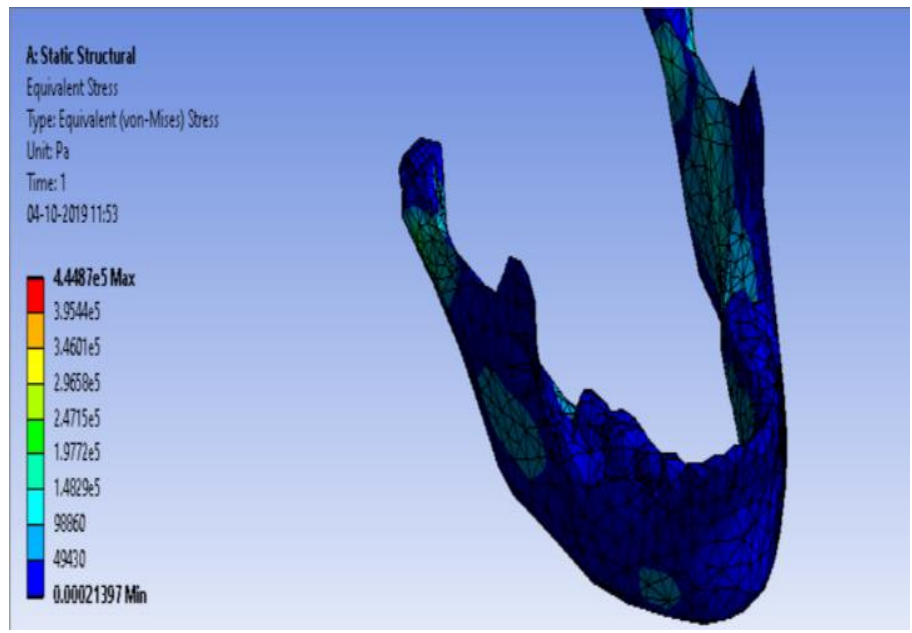

(a) Normal jaw (Balanced Occlusion)

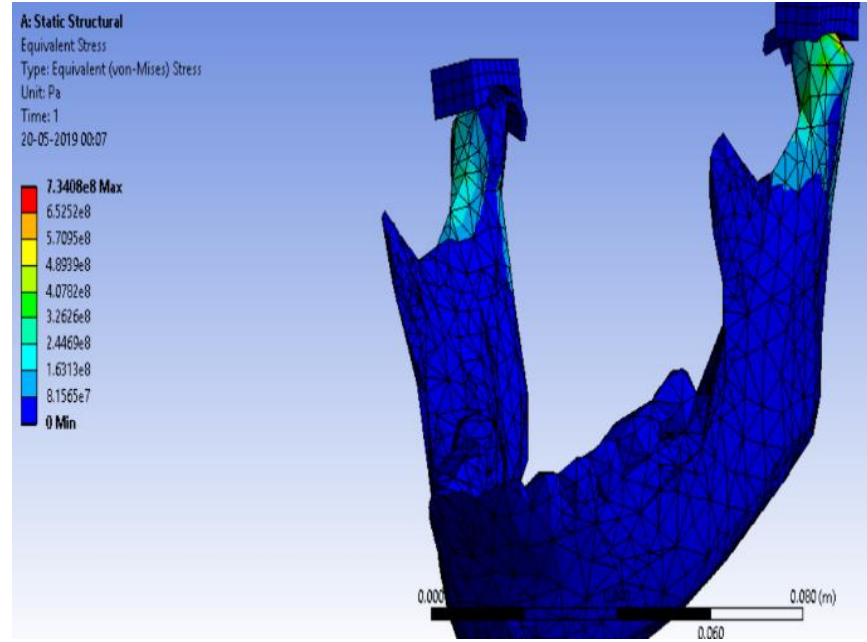

(b) Fused Jaw (Balanced Occlusion)

Figure 7. Balanced occlusion

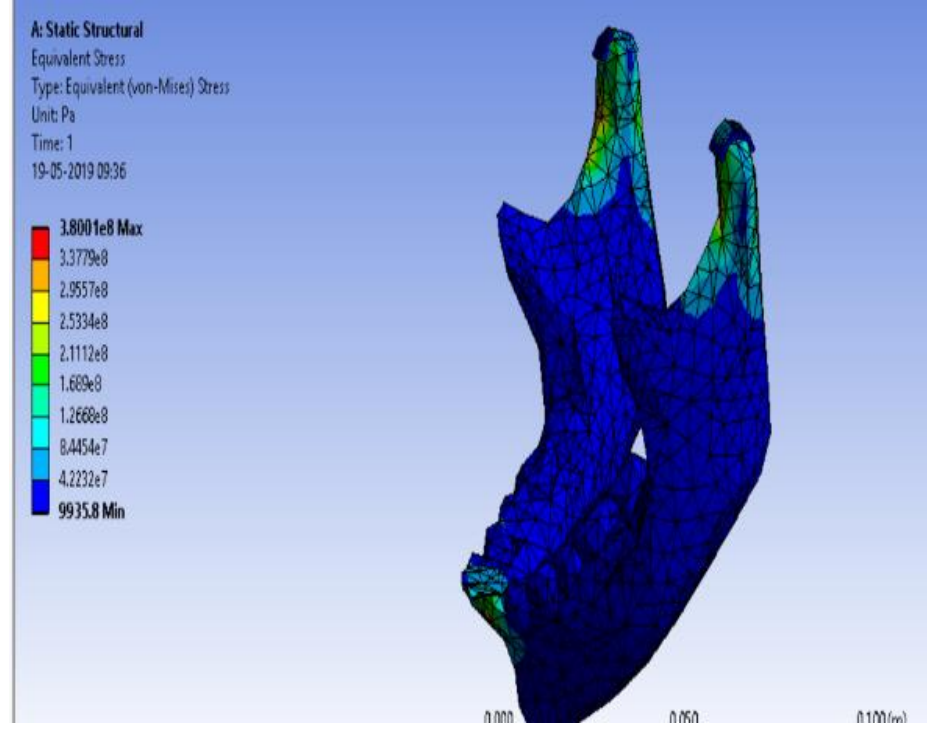

(a) Normal jaw (Bruxism)

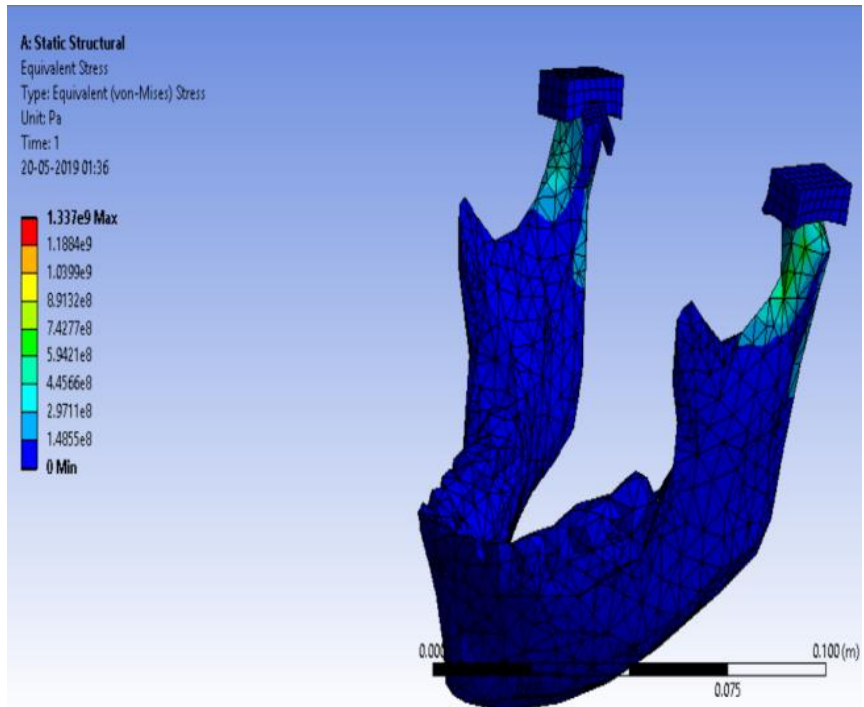

(b) Fused Jaw (Bruxism)

Figure 8. Bruxism

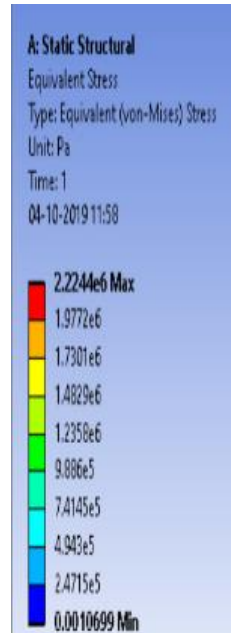

(a) Normal Jaw (Clenching)

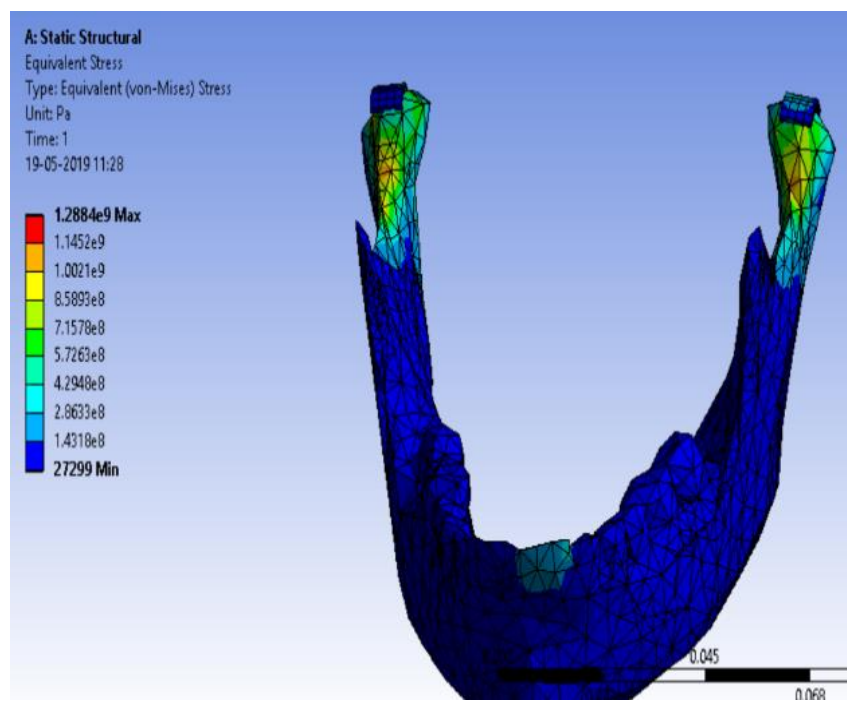

(b) Fused Jaw (Clenching)

Figure 9. Clenching 
In all loading conditions, it is observed that the maximum Von Mises stress occurs at the condylar head. FEA results as shown in the graph (Figure 10) concur that the least Von Mises stress happens during balanced occlusion. It is also observed that in increasing order, the maximum Von Mises stress is observed during unbalanced occlusion, bruxism, and clenching respectively as shown in Figure 11. In FEA simulation for a jaw with ankylosis, the maximum Von Mises stresses are quite higher.

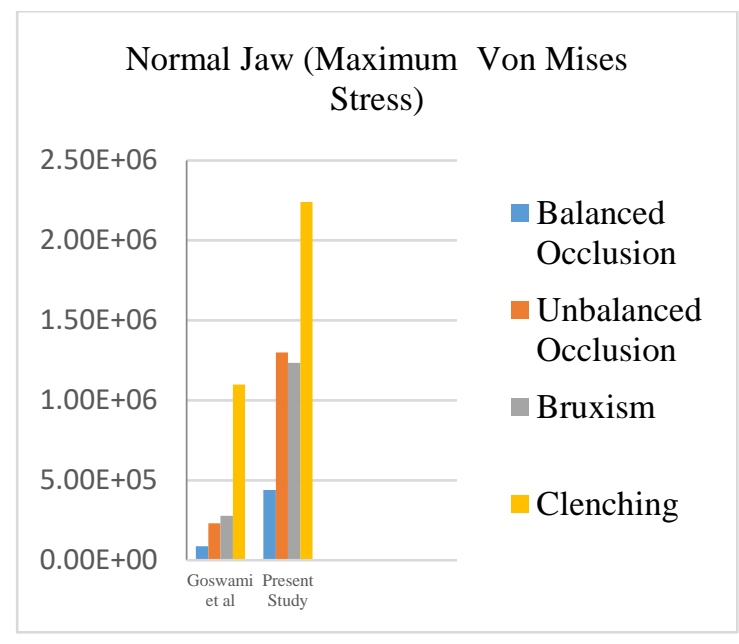

Figure 10. Maximum von Mises stress (Pa) during finite element simulation for four different load conditions

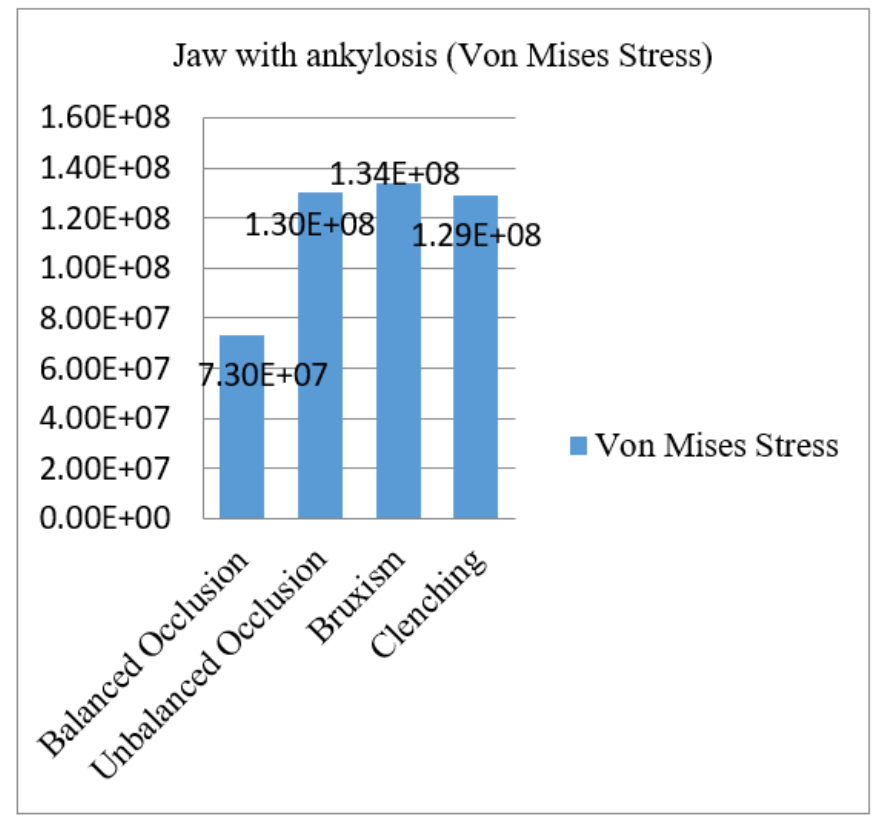

Figure 11. Maximum Von Mises Stress (Pa) for fused jaw (jaw with ankylosis)

\subsection{Discussion}

This paper aims to highlight the vitality of in vitro techniques like FEM or other analytical technologies for biomechanical evaluation of natural human temporomandibular joint to perceive its anatomy, stresses, structural and practical aspects. This paper aims at finding out the effects of varying load conditions on healthy TMJ vs. TMJ with ankylosis for the Indian populace.TMJ is the most extensively used load-bearing joint used in the human body and to establish the type and amount of stresses acting generally on this joint is extremely crucial as it will help in further design and development of a prosthesis for patients suffering from end-stage TM disorders.

Table 3. Comparative values of max Von Mises stress for normal jaw

\begin{tabular}{|c|c|c|c|c|}
\hline \multirow{2}{*}{$\begin{array}{c}\text { S } \\
\text { No. }\end{array}$} & \multirow{2}{*}{$\begin{array}{l}\text { Type of } \\
\text { loading }\end{array}$} & \multicolumn{3}{|c|}{$\begin{array}{c}\text { Maximum Von Mises Stress (Pa) in } \\
\text { Normal Human Jaw }\end{array}$} \\
\hline & & $\begin{array}{c}\text { Current } \\
\text { Study }\end{array}$ & $\begin{array}{c}\text { Base Paper } \\
{[20]}\end{array}$ & $\begin{array}{l}\text { Other } \\
\text { Studies }\end{array}$ \\
\hline 1. & $\begin{array}{l}\text { Balanced } \\
\text { Occlusion }\end{array}$ & $4.4 \mathrm{e}+5$ & $0.884 e+5$ & $0.35 \mathrm{e}+6$ \\
\hline 2. & $\begin{array}{l}\text { Unbalanced } \\
\text { Occlusion }\end{array}$ & $1.30 \mathrm{e}+6$ & $2.30 \mathrm{e}+5$ & \\
\hline 3. & Bruxism & $1.24 \mathrm{e}+6$ & $2.79 \mathrm{e}+5$ & \\
\hline 4. & Clenching & $2.24 \mathrm{e}+6$ & $1.10 \mathrm{e}+6$ & $1.68 \mathrm{e}+8$ \\
\hline
\end{tabular}

Von Mises stresses are reported for healthy TMJ and TMJ with ankylosis for four different loading conditions, i.e. normal loading, unbalanced occlusion, bruxism and clenching. As expected, clenching created high stresses around the disc surfaces and higher stresses are observed in TMJ with ankylosis in all the four loading conditions. Von Mises stresses corresponds to tissue strains rather than contact pressures, hence in the case of unhealthy TMJ, stresses are mostly higher as a disc with tissues have been displaced from its original position and bones are fused at condyle and fossa contact. The present study gives an all-inclusive investigation of all the loading conditions that have a compounding effect on the functioning of TMJ.

In this paper, the results of basic simulation tasks are compared to literature values from the past data.

1. The Von Mises stresses values observed for varying load conditions for a normal jaw in the present study as well as from literature are given in Table 3:

2. Von Mises stresses values for varying load conditions for a jaw with ankylosis are given in Table 4:

Table 4. Comparative values of max Von Mises stress for a jaw with ankylosis

\begin{tabular}{cccc}
\hline $\begin{array}{c}\text { S } \\
\text { No. }\end{array}$ & Type of loading & $\begin{array}{c}\text { Maximum Von Mises Stress (Pa) } \\
\text { in Jaw with Ankylosis } \\
\text { Current Study } \\
\text { Other Studies }\end{array}$ \\
\hline 1. & $\begin{array}{c}\text { Balanced } \\
\text { Occlusion }\end{array}$ & $7.3 \mathrm{e}+07$ & $8 \mathrm{e}+06$ \\
2. & $\begin{array}{c}\text { Unbalanced } \\
\text { Occlusion }\end{array}$ & $1.3 \mathrm{e}+08$ & - \\
3. & $\begin{array}{c}\text { Bruxism or Teeth } \\
\text { Grinding }\end{array}$ & $1.34 \mathrm{e}+08$ & - \\
4. & Clenching & $1.29 \mathrm{e}+08$ & $6.8 \mathrm{e}+08$ \\
\hline
\end{tabular}

\section{CONCLUSION}

1. The observations made for maximum Von Mises stress in the current study for normal jaw and jaw with ankylosis are compared and validated with available literature in Tables 3 and 4 respectively.

2. The approach under the current study allows patientspecific anatomical $3 \mathrm{~d}$ reconstruction of complex body structures and their functional constituents. The model allows for a dynamic investigation of the TMJ (healthy and disc displaced) and compares the type 
and number of stresses.

3. The FE models can successively assist in studying the biomechanical behavior of intricate structures which can further assist in improving the design, efficiency and durability of artificial prostheses.

4. Patient-specific 3D modeling can also help in designing customized artificial prosthetic devices for patients suffering from end-stage TMJ disorders with better fit, fixation, efficiency and long life. However, more dynamic as well as static analyses must be carried out along with experimental validation for a comprehensive investigation.

\section{ACKNOWLEDGMENT}

The present work is part of a DST project funded under the women scientist scheme WOS-A titled "Design and development of modular Temporomandibular joint prosthesis for patients suffering from end-stage TM disorders" (SR/WOS-A/ET-44/2017). The author is thankful to the Department of Science and Technology, Ministry of Science and Technology, New Delhi, India for funding the project and research work.

\section{REFERENCES}

[1] Gray, H. (1918). Anatomy of the Human Body, 20th ed Philadelphia: Lea \& Febiger.

[2] Spencer, M.A., Demes, B. (1993). Biomechanical analysis of masticatory system configuration in Neandertals and Inuits. American Journal of Physical Anthropology, 91(1): 1-20. https://doi.org/10.1002/ajpa.1330910102

[3] Chen, J., Xu, L. (1994). A finite element analysis of the human temporomandibular joint. Journal of Biomechanical Engineering, 116(4): 401-407. https://doi.org/10.1115/1.2895790

[4] Chen, J., Akyuz, U., Xu, L., Pidapart, R.M.V. (1998). Stress analysis of the human temporomandibular joint Medical Engineering Physics, 20: 565-572. https://doi.org/10.1016/S1350-4533(98)00070-8

[5] Breul, R., Mall, G., Landgraf, J., Scheck, R. (1999). Biomechanical analysis of stress distribution in the human temporomandibular-joint. Annals of Anatomy Anatomischer Anzeiger, 181(1): 55-60. https://doi.org/10.1016/S0940-9602(99)80090-9

[6] Tanaka, E., Rodrigo, D.P., Miyawaki, Y., Lee, K., Yamaguchi, K., Tanne, K. (2000). Stress distribution in the temporomandibular joint affected by anterior disc displacement: A three-dimensional analytic approach with the finite-element method. Journal of Oral Rehabilitation, 27(9): 754-759. https://doi.org/10.1046/j.1365-2842.2000.00597.x

[7] Tanaka, E., Rodrigo, D.P., Tanaka, M., Kawaguchi, A., Shibazaki, T., Tanne, K. (2001). Stress analysis in the TMJ during jaw opening by use of a three-dimensional finite element model based on magnetic resonance images. International Journal of Oral and Maxillofacial Surgery, 30(5): 421-430. https://doi.org/10.1054/ijom.2001.0132

[8] del Pozo, R., Tanaka, E., Tanaka, M., Kato, M., Iwabe, T., Hirose, M., Tanne, K. (2003). Influence of friction at articular surfaces of the temporomandibular joint on stresses in the articular disk: A theoretical approach with the finite element method. The Angle Orthodontist, 73(3): 319-327. https://doi.org/10.1043/00033219(2003)073<0319:IOFAAS > 2.0.CO;2

[9] Tanaka, E., del Pozo, R., Tanaka, M., Asai, D., Hirose, M., Iwabe, T., Tanne, K. (2004). Three-dimensional finite element analysis of human temporomandibular joint with and without disc displacement during jaw opening. Medical Engineering \& Physics, 26(6): 503-511. https://doi.org/10.1016/j.medengphy.2004.03.001

[10] Kober, C., Erdmann, B., Lang, J., Sader, R., Zeilhofer, H.F. (2004). Adaptive finite element simulation of the human mandible using a new physiological model of the masticatory muscles. Proceedings in Applied Mathematics and Mechanics, 4(1): 332-333. https://doi.org/10.1002/pamm.200410147

[11] Strait, D.S., Wang, O., Dechow, P.C., Ross, C.F., Richmond, B.G., Spencer, M.A., Patel, B.A. (2005). Modeling elastic properties in finite-element analysis: How much precision is needed to produce an accurate model. The Anatomical Record, 283A(2): 275-287. https://doi.org/10.1002/ar.a.20172

[12] Ren, S., Li, Q., Rong, Q. (2012). Finite element analysis of asymptomatic TMJ disc during mouth opening. System Simulation and Scientific Computing. ICSC 2012. Communications in Computer and Information Science, vol 326. Springer, Berlin, Heidelberg. https://doi.org/10.1007/978-3-642-34381-0_49

[13] Milne, N., O'Higgins, P. (2013). Applying geometric morphometrics to compare changes in size and shape arising from finite element analyses. Hystrix, 24(1): 126132. https://doi.org/10.4404/hystrix-24.1-6284

[14] Richmond, B.G., Wright, B.W., Grosse, I., Dechow, P.C., Ross, C.F., Spencer, M.A., Strait, D.S. (2005). Finite element analysis in functional morphology. The Anatomical Record, 283A(2): 259-274. https://doi.org/10.1002/ar.a.20169

[15] Bourke, J., Wroe, S., Moreno, K., McHenry, C., Clausen, P. (2008). Effects of gape and tooth position on bite force and skull stress in the dingo (Canis lupus dingo) using a 3 Dimensional finite element approach. PLOS ONE, 3(5): e2200. https://doi.org/10.1371/journal.pone.0002200

[16] Moreno, K., Wroe, S., Clausen, P., McHenry, C., D’Amore, D.C., Rayfield, E.J., Cunningham, E. (2008). Cranial performance in the Komodo dragon (Varanus komodoensis) as revealed by high-resolution 3-D finite element analysis. Journal of Anatomy, 212(6): 736-746. https://doi.org/10.1111/j.1469-7580.2008.00899.x

[17] Aoun, M., Mesnard, M., Ramos, A., Morlier, J., Puel, F. Cid, M. (2010). 2D-finite element models of the TMJ in three different mandible positions, simulation of clenching. Computer Methods in Biomechanics and Biomedical Engineering, 13(S1): 15-17. https://doi.org/10.1080/10255842.2010.490089

[18] Mesnard, M., Ramos, A., Ballu, A., Morlier, J., Cid, M., Simoes, J.A. (2011). Biomechanical analysis comparing natural and alloplastic temporomandibular joint replacement using a finite element model. J. Oral Maxillofac Surg., 69(4): 1008-1017. https://doi.org/10.1016/j.joms.2010.02.019

[19] Koolstra, J.H. (2011). Biomechanical analysis of the influence of friction in jaw joint disorders. Osteoarthritis $\begin{array}{lll}\text { and } & \text { Cartilage, } & \text { 20(1): }\end{array}$ 
https://doi.org/10.1016/j.joca.2011.10.009

[20] Cox, P.G., Fagan, M.J., Rayfield, E.J., Jeffery, N. (2011). Finite element modelling of squirrel, guinea pig and rat skulls: Using geometric morphometrics to assess sensitivity. Journal of Anatomy, 219(6): 696-709. https://doi.org/10.1111/j.1469-7580.2011.01436.x

[21] Dimitroulis, G. (2012). Temporomandibular joint surgery: What does it mean to India in the 21 st century? Journal of Maxillofacial and Oral Surgery, 11(3): 249257. https://doi.org/10.1007/s12663-012-0419-x

[22] Ingawale, S., Goswami, T. (2012). Biomechanics of the Temporomandibular https://doi.org/10.5772/33702

[23] Creuillot, V., Areiza, D., Sapin-de Brosses, E., Bonnet, A.S., Lipinski, P. (2013). Finite element analysis of temporomandibular joints during opening-closing motion: Asynchronous case report. Computer Methods in Biomechanics and Biomedical Engineering, 16(S1): 300-301. https://doi.org/10.1080/10255842.2013.815910

[24] Abe, S., Kawano, F., Kohge, K., Kawaoka, T., Ueda, K., Hattori-Hara, E., Mori, H., Kuroda, S., Tanaka, E. (2013). Stress analysis in human temporomandibular joint affected by anterior disc displacement during prolonged clenching. Journal of Oral Rehabilitation, 40(4): 239-246. https://doi.org/10.1111/joor.12036

[25] Li, Q., Ren, S., Ge, C., Sun, H.Y., Lu, H., Duan, Y.Z., Rong, Q.G. (2014). Effect of jaw opening on the stress pattern in a normal human articular disc: Finite element analysis based on MRI images. Head \& Face Medicine, 10: 24. https://doi.org/10.1186/1746-160X-10-2

[26] Duarte, R.J., Ramos, A., Mesnard, M. (2014). The influence of disc wear on the behavior of the temporomandibular joint: a finite element analysis in a specific case. Advances in Biomechanics and Applications, 1(3): 159-167. https://doi.org/10.12989/ABA.2014.1.3.159
[27] Mhamad, A., Antonio, R., Michel, M. (2015). Experimental and numerical study of the biomechanics of a human joint. 2015 International Conference on Advances in Biomedical Engineering (ICABME), Beirut, pp. 230-233. https://doi.org/10.1109/ICABME.2015.7323294

[28] Sun, M.X., Yang, J.J., Zhou, R.Z., Li, N.Y., Xia, J.N., Gu, F. (2015). Mechanical analysis on individualized finite element of temporal-mandibular joint under overlarge jaw opening status. International Journal of Clinical and Experimental Medicine, 8(6): 9046-9054.

[29] Ndukwe, E., Anitha, G. (2016). The human jaw movement analysis by finite element analysis. 2016 International Conference on Communication and Signal Processing (ICCSP), Melmaruvathur, India, pp. 06190622. https://doi.org/10.1109/ICCSP.2016.7754214

[30] Ren, S., Li, Q.H., Ge, C., Sun, H.Y., Lu, H., Duan, Y.Z., Rong, Q.G. (2016). MRI-based analysis of stress trajectories in the articular disc during jaw opening. International Journal of Clinical and Experimental Medicine, 9(2): 927-935.

[31] Tsouknidas, A., Jimenez-Rojo, L., Karatsis, E., Michailidas, N., Mitsiadis, T.A. (2017). A biorealistic finite element model to evaluate the effect of masticatory loadings on mouse mandible-related tissues. Frontiers in Physiology, 8 : 273. https://doi.org/10.3389/fphys.2017.00273

[32] Gomes, S., Ângelo, D.F., Pascoal-Faria, P., Mateus, A., Alves, N. (2018). Finite element analysis of a human temporomandibular joint disc: Preliminary result. AIP Conference Proceedings, 1978(1): 160004. https://doi.org/10.1063/1.5043814

[33] Stansfield, E., Parker, J., O'Higgins, P. (2018). A sensitivity study of human mandibular biting simulations using finite element analysis. Journal of Archaeological Science: $\quad$ Reports, 22: 420-432. https://doi.org/10.1016/j.jasrep.2018.04.026 